\title{
A EFETIVIDADE DA TERAPIA DE EXPOSIÇÃO PARA TRATAMENTO DO TRANSTORNO DE ESTRESSE PÓS-TRAUMÁTICO
}

\author{
Letícia Galery Medeiros \\ Christian Haag Kristensen
}

\author{
Programa de Pós-Graduação em Psicologia \\ Pontifícia Universidade Católica do Rio Grande do Sul (PUCRS) \\ Endereço eletrônico: legalery@yahoo.com
}

\begin{abstract}
Resumo
O Transtorno de Estresse Pós-Traumático (TEPT) é um transtorno de ansiedade que se desenvolve em indivíduos após a exposição a um evento estressor traumático, como violência interpessoal, acidentes ou desastres naturais. A psicoterapia cognitivo-comportamental (TCC) é o tratamento de escolha para este transtorno podendo envolver diferentes componentes, tais como psicoeducação, reestruturação cognitiva ou exposição. A terapia de exposição possui seus fundamentos na teoria do processamento emocional e pode ser empregada tanto como um componente da TCC quanto como um tratamento único. Este ensaio visa discutir a efetividade da terapia de exposição no TEPT. Inicialmente, serão apresentadas as teorias que embasam os tratamentos cognitivo-comportamentais para o TEPT. Após, serão revisadas as modalidades de exposição para o TEPT e por fim, são consideradas as limitações do tratamento com exposição. Conclui-se que apesar da terapia de exposição não ser efetiva para todos os tipos de pacientes e nem para todos os tipos de traumas, pode ser considerada o tratamento de escolha para o TEPT, apresentando elevado apoio empírico.
\end{abstract}

Palavras-chave: transtorno de estresse pós-traumático, terapia de exposição, terapia cognitivocomportamental. 


\section{Exposição a trauma e o desenvolvimento do transtorno de estresse pós-traumático}

Em 1980, o transtorno de estresse pós-traumático (TEPT) foi incluído na classificação oficial do manual de transtornos mentais, o DSM - III (APA, 1980). Este fato marcou o início das pesquisas contemporâneas sobre as consequências psiquiátricas da exposição a traumas. Ao longo da vida, cerca de metade dos norteamericanos serão expostos a pelo menos um evento traumático - tais como agressão, combate militar, acidente industrial ou automobilístico, estupro, violência doméstica ou desastre natural (FRIEDMAN, 2009). A maioria das pessoas consegue absorver o impacto psicológico de tal experiência e retomar uma vida normal; porém, não é o que ocorre para um significativo número de pessoas. Achados de um levantamento nacional sobre comorbidades (BRESLAU; KESSLER, 2001; KESSLET et al.,1995) indicam que 68,1\% a 89,6\% dos indivíduos teriam experienciado um evento de intensidade suficiente para eliciar sintomas de TEPT. Entre os expostos a trauma, 5\% a 6\% dos homens e $10 \%$ a $14 \%$ das mulheres desenvolvem TEPT (BRESLAU et al.,1998). O TEPT está entre os transtornos mais prevalentes, e apresenta consequências graves aos afetados.

O TEPT apresenta um quadro de sintomas ou deficiências do funcionamento diário associado ao trauma com duração de pelo menos um mês e, às vezes, perdura por toda a vida. Consiste de três grupos de sintomas - revivência, evitação/entorpecimento e hipervigilância - e de critérios para persistência e gravidade; podendovariar de leve à grave. Esses sintomas refletem a persistência de pensamentos, sensações e comportamentos especificamente relacionados ao evento traumático (FRIEDMAN, 2009). Há pacientes que atingem a recuperação total; outros, apenas parcial e alguns nunca se recuperam. O curso de longo prazo para a maioria das pessoas com TEPT crônico é marcado por remissões e recidivas. Há três classes gerais de portadores de TEPT: TEPT crônico, TEPT em remissão com recidivas ocasionais e de início tardio (ROTHBAUM et al., 2000; TAYLOR, 2006). Por estas razões, é particularmente importante identificar tratamentos efetivos.

\section{Teorias sobre o TEPT}

As teorias sobre o TEPT, apresentadas a seguir, oferecem uma visão sobre a origem dos sintomas e sua relação com o evento 
traumático, buscam demonstrar uma compreensão sobre o curso dos sintomas e a eficácia das técnicas cognitivo-comportamentais na redução dos mesmos.

Asteorias contemporâneas daaprendizagem(dessembilização sistemática, treino de relaxamento, biofeedback) tentam dar conta de muitos dos sintomas de desenvolvimento e manutenção do TEPT (ROTHBAUM et al., 2000). Os sintomas de revivência e excitabilidade são vistos como respostas emocionalmente condicionadas que resultam de um condicionamento clássico, sendo eliciadas por estímulos do ambiente. De acordo com a Teoria Comportamental, embora os sintomas iniciais possam ter sido causados pelo trauma, alguns sintomas atuais podem representar tentativas de manejar o sofrimento decorrente do trauma. Essas tentativas, no entanto, respondem a contingências situacionais atuais e tornam-se funcionalmente autônomas. Os comportamentos de evitação estão sob controle operante; porém os reforçadores ambientais apropriados podem estar faltando, podem ser ineficazes ou aversivos. Os comportamentos problemáticos estão sob controle dos estímulos antecedentes e dos estímulos reforçadores que afetam a probabilidade de ocorrência do comportamento (KEANE et al., 1985). Pensamentos, sentimentos e respostas psicológicas são classificados como eventos particulares que podem servir tanto como estímulos antecedentes quanto como consequências. Portanto, como resultado do emprego da análise comportamental, o foco do tratamento pode não ser necessariamente o trauma em si, mas os comportamentos desadaptativos que surgiram após o trauma. Contudo, a hipótese é de que a exposição a estímulos condicionados na ausência de consequências negativas extinguiria as reações emocionais condicionadas. Logo, para a Teoria Comportamental a exposição é presumida como o tratamento apropriado para os sintomas de revivência e excitabilidade, enquanto o manejo das contingências poderia ser implementado para os problemas de evitação e outros problemas comportamentais (ROTHBAUM et al., 2000).

A Teoria do Processamento Emocional (FOA; KOZAK, 1986) assegura que o TEPT emerge a partir do desenvolvimento de uma estrutura patológica do medo sobre o evento traumático. Essa estrutura inclui representações sobre o estímulo, as respostas e seus significados. Desta forma, qualquer informação associada ao trauma ativaria a estrutura do medo. É hipotetizado que a estrutura do medo em pessoas com TEPT possui um grande 
número de elementos de estímulos, sendo assim facilmente ativada. As tentativas de evitar essa ativação resultariam nos sintomas de evitação do TEPT. A Teoria do Processamento Emocional propõe que o sucesso da terapia envolve corrigir os elementos patológicos da estrutura do medo, e essa correção consiste no elemento essencial do processamento emocional. Desta forma, duas condições são necessárias para a redução do medo. Primeiro, a estrutura do medo precisa ser ativada. Segundo, novas informações precisam ser oferecidas, incluindo elementos incompatíveis com os elementos psicopatológicos, para que esses possam ser corrigidos. Nesta abordagem os procedimentos de exposição visam auxiliar o paciente a confrontar os materiais relacionados ao trauma, ativando a memória do trauma. Essa ativação constituise em uma oportunidade de integrar informações corretivas, e então modificar os elementos patológicos da memória traumática (ROTHBAUM et al., 2000).

Foa e Rothbaum (1998) propõem vários mecanismos que estariam envolvidos nas mudanças específicas relevantes para a melhoria do TEPT. Primeiramente, repetidas revivências imaginárias do trauma, através do uso das técnicas de exposição, promoveriam a habituação e assim reduziriam a ansiedade antecipatória associada à memória traumática. Também oportunizariam a correção da concepção equivocada de que a ansiedade continuaria para sempre até que a evitação ou fuga fossem realizadas. Segundo, o processo de deliberadamente confrontar a memória traumática bloquearia a conexão de reforçamento negativo de que há redução do medo quando há evitação cognitiva dos pensamentos e sentimentos associados ao trauma. Terceiro, reviver o trauma em um ambiente terapêutico e acolhedor incorporaria informações sobre segurança às memórias traumáticas, auxiliando o paciente a dar-se conta de que recordar o trauma não é perigoso. Quarto, focalizar a memória do trauma por um período prolongado de tempo ajuda o paciente a diferenciar o evento traumático de outros eventos não-traumáticos. Esse processo facilita a interpretação do trauma como uma ocorrência específica em vez de uma representação do mundo como um lugar perigoso e de si mesmo como incompetente. Quinto, o processo de revivência imaginária favorece a mudança de significados dos sintomas do TEPT, de um sinal de incompetência pessoal para uma sensação de controle e coragem. Sexto, revivências repetidas e prolongadas do evento traumático permitem a oportunidade de 
focar nos detalhes centrais das avaliações negativas de si mesmo e modificá-las. Todos estes mecanismos apresentados aqui também ocorrem nas exposições in vivo (ROTHBAUM et al., 2000, p. 62).

Por fim, numa tentativa de conciliar as teorias do TEPT, Brewin, Dalgleish e Joseph (1996) propõem a Teoria da Representação Dual que incorpora a Teoria do Processamento Emocional e a Teoria Social-Cognitiva. Eles propõem que a entrada sensorial está sujeita tanto a processos conscientes como inconscientes. A Teoria da Representação Dual descreve dois tipos de reações emocionais. Um dos tipos de reação emocional, condicionada durante o evento (por exemplo, medo, raiva), é ativado junto com a revivência das informações sensoriais e fisiológicas. As outras emoções (culpa, vergonha, tristeza) seriam secundárias, resultando das consequências e implicações do trauma. Os autores propõem que o processamento emocional do trauma possui dois elementos: a ativação das memórias não-conscientes (como sugerido pelas teorias do processamento cognitivo) e as tentativas conscientes para encontrar significado ou sentido, para atribuir causalidade ou responsabilidade, e para resolver conflitos entre o evento e as expectativas ou crenças prévias. $\mathrm{O}$ propósito deste processo é reduzir as emoções negativas e restaurar um relativo senso de segurança e controle sobre o ambiente. Esta teoria sugere que tanto a exposição como a terapia cognitiva podem ser necessárias como intervenção psicoterápica (ROTHBAUM et al., 2000).

Logo, na Teoria Comportamental assim como nas Teorias do Processamento da Informação, a exposição é presumida como o tratamento apropriado para os sintomas de revivência (memória) e excitabilidade, enquanto o manejo das contingências seria implementado para a evitação e outros problemas comportamentais (FOA; KEANE; FRIEDMAN, 2004).

\section{Psicoterapia Cognitivo-Comportamental para o TEPT}

A psicoterapia cognitivo-comportamental (TCC) para o TEPT objetiva modificar as cognições e os comportamentos desenvolvidos como uma resposta ao trauma, os quais se presumem que também estejam envolvidos na manutenção do TEPT. O tratamento enfatiza uma aliança colaborativa, por esse motivo, rotineiramente tem início com uma educação sobre o modelo cognitivo-comportamental e apresenta uma argumentação 
profunda sobre a justificativa do tratamento. Tipicamente o propósito do tratamento é a modificação dos comportamentos de evitação e dos pensamentos irrealísticos ou desadaptativos, que de acordo com o modelo da TCC são os fatores chaves da manutenção do TEPT (FOA; KEANE; FRIEDMAN, 2004). Foram desenvolvidas uma série de variações da TCC para o TEPT. As diferentes formas de tratamento podem ser nomeadas de acordo com seus componentes principais (por exemplo, reestruturação cognitiva ou exposição), ou pode-se referir a elas por seus nomes específicos, tais como: terapia do processamento cognitivo (RESICH: SCHNICKE, 1992) ou exposição prolongada (FOA: ROTHBAUM, 1998). A maioria das formas de TCC para o TEPT consiste em três elementos principais, variando na ênfase dada a cada um deles: psicoeducação, exposição e reestruturação cognitiva (ZAYFERT; BECKER, 2007).

\section{Terapia de Exposição}

A técnica de exposição a estímulos temidos foi desenvolvida por Meyer, nos anos 60, e superou as técnicas de dessensibilização e de relaxamento para o tratamento da ansiedade fóbica e dos rituais compulsivos (SALKOVSKIS, 1999). A técnica consiste na exposição direta do paciente aos estímulos temidos ou às situações temidas e evitadas por serem desencadeadoras de ansiedade. A exposição é feita repetidamente, de forma abrupta ou gradual, ao vivo ou imaginativa, conforme mais indicado pela avaliação dos sintomas e do contexto (RICHARD; LAUTERBACH, 2007). O tempo de exposição deve ser longo o bastante para permitir o aumento crescente da ansiedade até um máximo e depois sua redução (FOA; KOSAK, 1986; MARSHALL, 1985). Esse tempo de permanência do paciente exposto ao estímulo viabiliza os processos de habituação e extinção. A habituação é um processo elementar de aprendizagem, no qual uma resposta, aprendida ou não, enfraquece após ser emitida repetidamente em um determinado contexto. Em outras palavras, o organismo deixa de responder diferencialmente a determinado estímulo porque se “acostuma” com ele (TAULOR, 2006). A extinção, por outro lado, ocorre devido à repetida exposição do organismo a uma situação na qual a presença de um estímulo ou a consequência aversiva não ocorre de fato (LAUTERBACH; REILAND, 2006). Se o paciente for afastado da situação ansiógena, ou se o estímulo for removido durante o período de aumento ou de pico da ansiedade, 
pode ocorrer o reforçamento do comportamento de fuga da situação ansiógena, devido à consequente terminação do evento e alívio da tensão.

A exposição abrupta pode ocorrer por implosão ou inundação por meio de imagens ou ao vivo. Nessa técnica, o paciente é exposto diretamente ao estímulo em toda a sua dimensão, ao vivo ou por imaginação, na forma provocativa da ansiedade mais intensa, sem interrupção, até que a ansiedade diminua (TAYLOR, 2006). O tempo de exposição varia na proporção inversa da intensidade da ansiedade. A partir de um determinado ponto, um intervalo de tempo $\mathrm{x}$, no qual a ansiedade sobe devido à presença do estímulo, ela começa a baixar pelos processos de extinção e de habituação. $\mathrm{Na}$ exposição protegida, é utilizada a dessensibilização sistemática, feita por imaginação e em pequenos passos, gradualmente e mantendo o paciente relaxado; ou ao vivo, com exposição programada a estímulos da vida real, também de modo gradativo. A exposição por imagens pode ser transferida posteriormente para as mesmas situações ao vivo, servindo como ensaio ou treino para o cliente. É importante que o tempo de exposição seja suficiente para que a ansiedade diminua antes que o paciente seja removido da situação, ou que o estímulo ansiógeno seja terminado (GUIMARÃES, 2001).

A exposição imagística envolve a exposição sistemática, repetida e prolongada às memórias traumáticas, incluindo as memórias após o trauma, se estas também provocarem sofrimento. Este tipo de exposição reduz o sofrimento associado às memórias traumáticas e à revivência dos sintomas. A exposição imagística também ensina ao paciente que as memórias do trauma e as emoções associadas a ele não são perigosas. Pode ser feita através de uma descrição por escrito do evento ou gravação da narrativa do mesmo (ROTHBAUM et al., 2000; TAYLOR, 2006).

A exposição interoceptiva envolve uma exposição sistemática aos elementos evocadores de medo, mas demonstrando que as sensações corporais são inofensivas. É uma promissora intervenção para o TEPT. O objetivo da exposição interoceptiva é reduzir a sensibilidade à ansiedade (medo ou evitação das sensações corporais), que ocupa um papel central na ampliação das reações de ansiedade e está diretamente envolvida com a sintomatologia do TEPT (LAUTERBACH; REILAND, 2006; TAYLOR, 2006).

A exposição situacional (in vivo ou in virtuo) envolve a exposição segura a um estímulo externo inofensivo que pareça ou relembre o paciente do trauma. A escolha do estímulo é 
determinada principalmente pelos objetivos do paciente com respeito aos estímulos geradores de sofrimento, os quais ele deseja superar. Os estímulos usados na exposição situacional incluem lugares, situações, objetos associados ao trauma e objetos simbólicos (TAYLOR, 2006).

\section{Terapia de Exposição e o Transtorno de Estresse Pós-Traumático}

O alvo da exposição no tratamento do TEPT é a evitação, onde busca-se encorajar o paciente a aproximar-se do estímulo temido, demonstrando que um estímulo seguro (embora temido) não precisa ser evitado. Durante a exposição, o paciente irá aproximar-se do estímulo por longos períodos de tempo para alcançar uma diminuição do medo (habituação) e repetidas vezes para promover uma redução persistente do medo (FOA; KEANE; FRIEDMAN, 2004).

As terapias comportamentais estiveram entre as primeiras intervenções utilizadas para o tratamento do TEPT e que demonstraram efetividade. Atualmente elas permanecem como um componente central dentro dos mais importantes tratamentos para oTEPT(CLOITRE,2009).Embora o cerne das intervenções foque as experiências traumáticas dos pacientes e a sintomatologia do TEPT, essas intervenções - particularmente a reestruturação cognitiva - podem auxiliar o paciente a lidar melhor com os estressores diários da vida. Os exercícios de regulação emocional também são utilizados para auxiliar os pacientes a tolerar e concluir os exercícios de exposição. O domínio e o prazer com os exercícios e as intervenções pessoais podem reforçar o nível de suporte social do paciente, que é muito importante no enfrentamento do estresse em geral e no sofrimento associado com a terapia de exposição. A aplicabilidade geral das intervenções da TCC para o enfrentamento dos estressores diários é importante, pois as pessoas com TEPT tendem a ser mais reativas aos estressores do que as pessoas sem o transtorno; isto é, o TEPT está associado a uma maior, mais longa e duradoura reação de excitabilidade fisiológica e emocional em resposta a estressores (TAYLOR, 2006).

As intervenções de exposição para o TEPT estavam focadas inicialmente em estímulos do ambiente tais como: sons, objetos ou lugares que recordassem o trauma. Entretanto, estudos experimentais com veteranos de combate que desenvolveram TEPT revelaram que simplesmente imaginar ou pensar sobre o 
trauma era o suficiente para eliciar as reações clássicas de medo (PITMAN et al., 1987). Estes estudos sugerem que um potente estímulo eliciador do medo pertence ao mundo interno do sujeito e que o "objeto" temido verbalizado representa a memória traumática do indivíduo (CLOITRE, 2009). De acordo, as intervenções de exposição foram expandidas para incluir não somente os estímulos externos (exposição in vivo) mas as recordações individuais do trauma (exposição imagística) (RIGGS; CAHILL; FOA, 2006).

Quando o paciente está pronto para a terapia de exposição, essa é implementada gradualmente, e tradicionalmente apenas um tipo de exercício de exposição (imagística, interoceptiva ou situacional) é conduzido por sessão. Entretanto, mais de uma forma de exposição pode ser implementada durante a semana como tarefa de casa (por exemplo: uma combinação de exercícios de exposição imagística e situacional). Quando é feito o planejamento de uma terapia de exposição, é importante que seja esclarecido ao paciente ou aos seus familiares qualquer concepção errônea ou equivocada que venham a ter sobre esta abordagem. $\mathrm{O}$ paciente e outras pessoas relevantes a ele precisam ser esclarecidos de que (1) a exposição é uma das intervenções mais efetivas para o TEPT, (2) a exposição é implementada gradualmente, usualmente junto com o desenvolvimento de estratégias de enfrentamento, e somente quando o paciente sente-se pronto, (3) a exposição é geralmente muito segura e não envolve objetivamente exposição a situações de risco, e (4) a exposição, assim como outros tratamentos, possui alguns efeitos colaterais, mas que tendem a ter curta duração (por exemplo: aumentos transitórios da ansiedade e pesadelos) (TAYLOR, 2006). Segundo Taylor (2006) a taxa de abandono de terapias com exposição (utilizada como única intervenção) não é diferente das taxas de abandono dos outros tratamentos para o TEPT que não utilizam a exposição.

A terapia de exposição é uma abordagem empiricamente validada para o tratamento do TEPT, mas não tem sido amplamente utilizada na prática clínica privada. Um estudo sobre a prática padrão no Veterans Administration Medical Center (ROSEN et al., 2004) encontrou que menos de $20 \%$ dos especialistas em TEPT conduziam tratamentos baseados na exposição, e menos de 10\% o fazem com regularidade. Esta percentagem não aumentou após a International Society for Traumatic Stress Studies (ISTSS) ter publicado seu guia prático que sustentava o uso das TE (ROSEN et al., 2004). 
O que se segue é a descrição da forma mais comum onde a terapia de exposição é praticada. Esta descrição consta do protocolo de Processamento Emocional (PE) descrito por Foa et al. (1999). Este protocolo é extremamente bem articulado e é o estudo mais abrangente sobre tratamentos com exposição.

O referido protocolo lista os componentes do tratamento em 10 sessões do protocolo de tratamento da PE. As sessões duram aproximadamente 90 minutos, e desde o princípio o foco do tratamento é o evento traumático. A exposição imaginária e in vivo são os dois elementos centrais do tratamento. A exposição imagística inicia na terceira sessão e continua nas sessões restantes. Durante a exposição imaginária, os participantes são solicitados a descrever e visualizar, tão vividamente quanto possível, o trauma no presente. A visualização foca o trauma como um todo e inclui aqueles eventos que ocorreram imediatamente antes e depois do trauma. Os participantes são encorajados a manterem os olhos fechados e a fornecerem o máximo de detalhes possíveis para melhorar a visualização e potencializar o engajamento emocional. As tarefas de casa incluem ouvir em áudio a sessão uma vez por dia. Certamente, esta é uma experiência terapêutica muito exigente e intensa, e torna-se essencial que o cliente tenha um entendimento claro sobre os argumentos da exposição imaginária. Como uma regra geral, é útil estruturar a argumentação da exposição como uma oportunidade do paciente processar o trauma, organizar suas memórias, e trazer sentido à experiência (FOA et al., 1999).

\section{Evidências empíricas da Terapia de Exposição para o TEPT}

Muitos estudos têm apresentado a eficácia de medicamentos e da psicoterapia no tratamento do TEPT. Van Etten e Taylor (1998) examinaram a eficácia de todos os tratamentos para o TEPT (61 estudos incluindo farmacoterapia, psicoterapia e grupos controle). No geral, os tratamentos psicológicos e farmacológicos superam os controles, onde o EMDR (eye movement desensitization and reprocessing) e a terapia cognitivocomportamental foram identificados como mais efetivos que os outros tratamentos (VAN ETTEN; TAYLOR, 1998).

Estudos de meta-análise mais recentes têm focado na eficácia de tratamentos psicológicos específicos para o TEPT. Estes estudos demonstram que os tratamentos focados no trauma são mais eficazes (BISSON; ANDREW, 2007; BISSON et al., 
2007; SEIDLER; WAGNER, 2006). As melhores evidências sugerem que a psicoterapia focada no trauma é mais efetiva e deve ser considerada a primeira linha de tratamento para o TEPT (FOA et al., 2004).

Powers et al. (2010) realizaram um estudo de meta-análise para analisar a eficácia da terapia de exposição prolongada para o TEPT em relação a grupos controle. Os resultados demonstraram que a terapia de exposição prolongada apresentou resultados significativamente melhores que os grupos controle nas medidas do TEPT tanto ao final do tratamento quanto no follow-up. Por outro lado, os resultados não demonstraram diferenças significativas entre a terapia de exposição prolongada e outros tratamentos (Terapia Centrada no Cliente, EMDR).

Apesar da forte evidência sobre a efetividade da terapia de exposição no tratamento do TEPT (POWERS et al., 2010), esta permanece subutilizada na prática clínica (ROSEN et al., 2004). Foram identificadas algumas variáveis que podem estar influenciando a decisão clínica sobre o método de tratamento a ser escolhido. Dentre as variáveis relacionadas aos terapeutas está a falta de treinamento e de experiência com a exposição para o TEPT (VAN MINNEN; HENDRIKS; OLFF, 2010). Nesse sentido haveria a necessidade de um investimento em treinamento para terapeutas que atuam especificamente com intervenção a traumas. Também foram relatadas algumas dificuldades por parte dos terapeutas em utilizar as técnicas de exposição. Dentre estas cita-se a percepção de que a terapia de exposição provoca mais desconforto que outras abordagens, e a existência de uma inibição em abordar-se o trauma diretamente, temendo uma revitimização dos pacientes (VAN MINNEN; HENDRIKS; OLFF, 2010). As variáveis relacionadas aos pacientes também são importantes. Casos em que ocorrem co-morbidades e/ou preferência por parte dos pacientes em relação ao tipo de tratamento também influenciam a decisão dos terapeutas (VAN MINNEN; HENDRIKS; OLFF, 2010).

\section{Limitações}

O uso das técnicas de exposição deve ser evitado ou adiado se houverem indicações de que o paciente não apresenta condições de lidar com o desconforto associado à exposição. Este pode ser o caso dos pacientes que apresentem dificuldades em lidar com 
controle de impulsos, transtornos por uso de substâncias, ideação suicida ou comportamentos auto-lesivos (TAYLOR, 2006).

Alguns terapeutas, com frequência, mostram-se preocupados com a excitação emocional experienciada pelos sobreviventes a traumas quando tratados com a TE, podendo esta mostrar-se extremamente estressante ou até danosa (RIGGS; CAHILL; FOA, 2006). De fato, muitos pesquisadores clínicos têm demonstrado reservas sobre a segurança da TE no tratamento de alguns casos de TEPT (CLOITRE et al., 2002; KILPATRICK; BEST, 1984; PITMAN et al., 1991). Duas questões em relação à segurança, em particular, têm chamado atenção na literatura: a TE pode exacerbar os sintomas do TEPT ao invés de reduzilos; e embora os sintomas do TEPT possam ser aliviados, outros sintomas psicológicos (por exemplo: consumo de drogas, depressão, culpa) podem piorar. Entretanto, os estudos que apontam tais preocupações não apresentam grupos controle ou faltam dados que sustentem estas inferências (PITMAN et al., 1991; TARRIER et al., 1999).

Alguns sobreviventes a trauma são relutantes em confrontar as recordações do evento e a tolerar altos índices de ansiedade e o incremento temporário dos sintomas que eventualmente acompanham a exposição. Sendo assim, não é qualquer pessoa que pode se candidatar à exposição. Há alguma evidência de que a exposição não é efetiva para criminosos, especialmente pacientes nos quais a culpa é a emoção primária (PITMAN et al., 1991). Também há evidências de que indivíduos em que a emoção primária é a raiva (FOA et al., 1995) podem não se beneficiar da exposição tanto quanto os indivíduos que apresentam a ansiedade como resposta emocional primária.

\section{Considerações finais}

Comparando os números e tipos de tratamento que dão suporte para cada tipo de intervenção para o TEPT, a exposição possui a maioria dos estudos e o maior número de estudos controlados (ROTHBAUM et al., 2000). Os estudos apontados (PITMAN et al., 1991; TARRIER et al., 1999; TAYLOR, 2006) demonstram que as medidas que indicariam piora associadas à exposição são geralmente muito baixas e que a terapia de exposição não está associada a grandes riscos tanto quanto outras formas de terapia. Em síntese, as evidências são bastante persuasivas 
sobre a efetividade da TE para o tratamento do TEPT. $\mathrm{Na}$ verdade, nenhuma outra modalidade de tratamento possui tantas evidências sobre sua eficácia (ROTHBAUM et al, 2000).

\section{Referências}

AMERICAN PSYCHIATRIC ASSOCIATION. Diagnostic and Statistical Manual of Mental Disorders. 4. ed.. Whashington, DC: American Psychiatric press, 2002. 494p.

BISSON, Jonathan Ian; ANDREW, Martin. Psychological treatment of post-traumatic stress disorder (PTSD). Cochrane Database of Systematic Review, Issue 3, Art. No.: CD003388. DOI: 003310.001002/14651858.CD14003388.pub14651853.2007.

BISSON, Jonathan Ian; EHLERS, Anke; MATTHEWS, Rosa; PILLING, Stephen; RICHARDS, David; TURNER, Stuart. Psychological treatments for chronic post-traumatic stress disorder: Systematic review and meta-analysis. The British Journal of Psychiatry, v. 190, p. 97-104, 2007.

BRESLAU, Naomi; KESSLER, Ronald C.; CHILCOAT, Howard D.; SCHULTZ, Lonni R., DAVIS, Glenn C.; ANDRESKI, Patricia. Trauma and posttraumatic stress disorder in the community: the 1996 Detroit area survey of trauma. American Journal of Psychiatry, v. 55, n. 7, p. 626-632, july 1998.

BRESLAU, Naomi; KESSLER, Ronald C. The stressor criterion in DSM-IV posttraumatic stress disorder: an empirical investigation. Biological Psychiatry, v. 50, n. 9, p. 699-704, nov. 2001.

BREWIN, Chris R.; DALGLEISH, Tim; JOSEPH, Stephen. A dual representation theory of posttraumatic stress disorder. Psychological Review, v. 103, n. 4, p. 670-686, oct. 1996.

CLOITRE, Marylene; KOENEN, Karestan C.; COHEN, Lisa R.; HAN, Hyemee. Skills training in affective and interpersonal regulation followed by exposure: A phase-based treatment for PTSD related to childhood abuse. Journal of Consulting and Clinical Psychology, v. 70, n. 5, p. 1067-1074, oct. 2002.

CLOITRE, Marylene. Effective Psychotherapies for Posttraumatic Stress Disorder: A Review and Critique. CNS Spectrums, v. 14, n. 1, p. 32-43, jan. 2009. 
FOA, Edna B.; KOZAK, Michael J. Emotional processing of fear: exposure to corrective information. Psychological Bulletin, v. 99, n. 1, p. 2035, jan. 1986.

FOA, Edna B., DANCU, Constance V.; HEMBREE, Elizabeth A.; JAYCOX, Lisa H.; MEADOWS, Elizabeth A.; STREET, Gordon P. A comparison of exposure therapy, stress inoculation training, and their combination for reducing posttraumatic stress disorder in female assault victims. Journal of Consulting and Clinical Psychology, v. 67, n. 2, p. 194-200, apr. 1999.

FOA, Edna B.; KEANE, Terence M.; FRIEDMAN, Matthew J. Effective treatments for PTSD: practice guidelines from the International Society for Traumatic Stress Studies. New York: Guilford Press, 2004.

FOA, Edna B.; RIGGS, David S.; MASSIE, Elise D.; YARCZWER, Matthew. The impact of fear activation and anger on the efficacy of exposure treatment for PTSD. Behavior Therapy, v. 26, n. 3, p. 487-499, 1995.

FOA, Edna B.; ROTHBAUM, Barbara Olasov. Treating the trauma of rape. New York: Guilford Press, 1998. 287 p.

FRIEDMAN, Matthew. Transtorno de Estresse Agudo e PósTraumático. 4. ed. Porto Alegre: Artmed, 2009.142 p.

GUIMARÃES, Suely Sales. Técnicas Cognitivas e Comportamentais. In: RANGÈ, Bernard (Org.). Psicoterapias Cognitivo-Comportamentais: um diálogo com a psiquiatria. Porto Alegre: Artmed, 2001. p. 113-130.

KEANE, Terence M.; FAIRBANK, John A.; CADDELL, Juesta M.; ZIMERING, Rose T.; BENDER, Mary E. A behavioral approach to assessing and treating posttraumatic stress disorder in Vietnan veterans. In: FIGLEY, Charles R. (Ed.). Trauma and its wake. New York: Brunner/Mazel, 1985. p. 257-294.

KESSLER, Ronald C.; SONNEGA, Amanda; BROMET, Evelyn; HUGHES, Michael; NELSON, Christopher B. Posttraumatic stress disorder in the National Comorbidity Survey. Archives of General Psychiatry, v. 52, n. 12, p. 1048-1060, dec. 1995.

KILPATRICK, Dean G.; BEST, Connie L. Some cautionary remarks on treating sexual assault victims with implosion. Behavior Therapy, $\mathrm{v}$. 15, n. 4, p. 421-423, sept. 1984.

KRAKOW, Barry; HOLLIFIELD, Michael; JOHNSTON, Lisa; 
KOSS, Mary; SCHRADER, Ron; WARNER, Teddy D. et al. Imagery rehearsal therapy for chronic nightmares in sexual assault survivors with posttraumatic stress disorder: a randomized controlled trial. Journal of the American Medical Association, v. 286, n. 5, p. 537-545, 2001.

LAUTERBACH, Dean; REILAND, Sarah. Exposure Therapy and Post-Traumatic Stress Disorder. In: RICHARD, David C. S.; LAUTERBACH, Dean. (Eds.). Handbook of Exposure Therapies. New York: Elsevier, 2007. p. 127-152.

MARSHALL, William L. The effects of variable exposure in flooding therapy. Behavior Therapy, v. 16, n. 2, p. 117-135, mar. 1985.

PITMAN, Roger K.; ALTMAN, Bruce; GREENWALD, Evan; LONGPRE, Ronald E.; MACKLIN, Michael L.; POIRE, Roger E. et al. Psychiatric complication during flooding therapy for posttraumatic stress disorder. Journal of Clinical Consulting Psychiatry, v. 52, n. 1, p. 17-20, jan. 1991.

PITMAN, Roger K.; ORR, Scott P.; FORGUE, Dennis F.; JONG, Jacob de; CLAIBORN, James M. Psychophysiologic assessment of posttraumatic stress disorder imagery in vietnam combat veterans. Archive of General Psychiatry, v. 44, n. 11, p. 970-975, nov. 1987.

POWERS, Mark B.; HALPERN, Jacqueline M.; FERENSCHAK, Michael P.; GILLIHAN, Seth J.; FOA, Edna B. A meta-analytic review of prolonged exposure for posttraumatic stress disorder. Clinical Psychology Review, v. 30, n. 9, p. 635-641, aug. 2010.

REISICK, Patricia A.; SCHNICKE, Monica K. Cognitive processing therapy for sexual assault victims. Journal of Consulting and Clinical Psychology, v. 60, n. 5, p. 748-756, oct. 1992.

RICHARD, David C. S.; LAUTERBACH, Dean. Handbook of Exposure Therapies. New York: Elsevier, 2007. 464 p.

RIGGS, David S.; CAHILL, Shawn. P.; FOA, Edna B. Prolonged Exposure Treatment of Posstraumatic Stress Disorder. In: FOLLETTE, Victoria M.; RUZEK, Josef. I. Cognitive-Behavioral Therapies for Trauma. 2. ed. New York: Guilford Press, 2006. p. 65-95.

ROSEN, Craig S. et al. VA practice patterns and practice guidelines for treating posttraumatic stress disorder. Journal of Traumatic Stress, Deerfield, v. 17, n. 3, p. 213-222, june 2004.

ROTHBAUM, Barbara Olasov; MEADOWS, Elizabeth A.; REISIK, 
Patricia; FOY, David W. Behavioral Therapy. In: FOA, Edna B., KEANE, Terence. M.; FRIEDMAN, Matthew J. (Eds.). Effective Treatments for PTSD. New York: The Guilford Press, 2000. p. 60-83.

SALKOVSKIS, Paul. M. Understanding and treating obsessive-compulsive disorder. Behaviour Research and Therapy, v. 37, p. 29-52, july 1999.

SEIDLER, Guenter H.; WAGNER, Frank E. Comparing the efficacy of EMDR and trauma-focused cognitive-behavioral therapy in the treatment of PTSD: A meta-analytic study. Psychological Medicine, Cambridge, v. 36, n. 11, p. 1515-1522, june 2006.

TARRIER, Nicholas et al. A randomized trial of cognitive therapy and imaginal exposure in the treatment of chronic posttraumatic stress disorder. Journal of Consulting and Clinical Psychology, Washington (DC), v. 67, n. 1, p. 13-18, Feb. 1999.

TAYLOR, Steven. Clinician's guide to PTSD: a cognitive-behavioral approach. New York: The Guilford Press, 2006.337 p.

VAN ETTEN, Michelle L.; TAYLOR, Steven. Comparative efficacy of treatments for post traumatic stress disorder: A meta-analysis. Clinical Psychology \& Psychotherapy, v. 5, n. 3, p. 126-144, Sept. 1998.

VAN MINNEN, Agnes; HENDRIKS, Lotte; OLFF, Miranda. When do trauma experts choose exposure therapy for PTSD patients? A controlled study of therapist and patient factors. Behaviour Research and Therapy, v. 48, n. 4, p. 312-320, 2010.

ZAYFERT, Claudia; BECKER, Carolyn B. Cognitive-Behavioral Therapy for PTSD: a case formulation approach. New York: The Guilford Press, 2008. 252 p. 


\section{Abstract}

Posttraumatic Stress Disorder (PTSD) is an anxiety disorder which emerges after exposure to a stressful traumatic event such as interpersonal violence, accidents or natural disasters. Cognitive-behavioral therapy (CBT) is the elective treatment for this disorder; it includes different components as psycho education, cognitive restructuring, or exposure. Exposure therapy derives from emotional processing theory and can be employed as a component of CBT or as a single modality of treatment. This essay aims to discuss the effectiveness of exposure therapy to PTSD. First, will be presented theories that provide the basis for cognitive-behavioral treatments for PTSD. Then, the modalities of exposure for PTSD will be reviewed, and treatment limitations will be considered. We conclude that despite exposure therapy is not effective for every patient or every kind of traumas, it can be considered the treatment of choice for PTSD, as it shows high empirical support.

Keywords: posttraumatic stress disorder, exposure therapy, cognitive-behavioral therapy. 
